\title{
Note sur la relation entre l'indice de réfraction de la matière grasse du lait et la précision des dosages de matière grasse par l'appareil Milko-Tester
}

\author{
par \\ R. JEUNET et R. GRAPPIN \\ I.N.R.A. Station Expérimentale Laitière (39) Poligny
}

Dans un précédent article (Grappin et Jeunet, 1970), nous avons souligné que la précision des dosages de matière grasse du lait selon le procédé Milko-Tester dépend de certaines propriétés physiques et chimiques du lait. Haugaard et Pettinati (1959) et Walstra (1965 a et 1965 b) indiquent que ce sont essentiellement la dimension des globules gras, l'indice de réfraction du sérum et celui de la graisse, ainsi que la présence de micelles de caséine dans le lait qui influent sur la précision des dosages. Mais la technique utilisée par le MilkoTester, en particulier la dilution du lait avec du Versène et l'homogénéisation de la matière grasse, diminuent beaucoup l'influence de la plupart de ces facteurs. Il subsiste cependant une source importante de variation représentée par l'indice de réfraction de la matière grasse du lait.

Ce travail a pour but de montrer les relations qui existent entre les variations d'indice de réfraction de la matière grasse du lait et la précision des dosages de matière grasse par le MilkoTester.

\section{I. - MATERIEL ET METHODES}

\section{a) Nature des laits}

L'essai porte sur 104 laits individuels provenant de vaches à tous les stades de lactation et prélevés entre les mois de mars et juillet 1970 dans 6 étables du Jura. Le taux de matière grasse des laits varie entre 18 et $49 \mathrm{~g} / \mathrm{kg}$.

\section{b) Analyses}

1) Dosages de la matière grasse : La teneur en matière grasse des laits a été déterminée, d'une part au moyen de l'appareil MilkoTester M K III et d'autre part selon la méthode de référence RöseGottlieb (norme FIL. 1 : 1955). 
Tous les dosages ont été faits en double sur des laits additionnés de 0,1 p. 100 de $\mathrm{Cr}_{2} \mathrm{O}_{7} \mathrm{~K}_{2}$ et conservés au plus 8 jours à $4^{\circ} \mathrm{C}$. Durant toute la période d'essai, l'étalonnage du Milko-Tester a été vérifié à l'aide d'un lait de contrôles et du lait gélifié « Milko-Gel » fourni par le constructeur.

2) Mesure des indices de réfraction : Pour extraire la matière grasse, nous avons utilisé la méthode de Pont (1955). Cependant afin d'éliminer complètement l'eau ainsi que les résidus de butanol, la graisse a été placée pendant $45 \mathrm{mn}$ à l'étuve à $100^{\circ} \mathrm{C}$.

Cette technique a donné des résultats identiques à la méthode de Mulder (1940) mais elle présente l'avantage de pouvoir être utilisée sans difficulté dans le cas de lait conservé avec du dichromate de potassium.

Les mesures d'indice de réfraction $\left(\mathrm{n}_{\mathrm{v}}\right)$ sont faites avec un réfractomètre Zeiss.Abbe à une température de $40^{\circ} \mathrm{C}$.

La répétabilité des mesures est de $\pm 10^{-4}$.

\section{II. - RESULTATS ET DISCUSSION}

Les écarts entre les résultats fournis par le Milko-Tester et la méthode Röse-Gottlieb sont donnés en différences relatives (en p. 100) par rapport aux résultats Röse-Gottlieb.

La figure 1 montre qu'il existe une liaison linéaire étroite entre les écarts Milko-Tester-Röse-Gottlieb et l'indice de réfraction de la matière grasse. La valeur du coefficient de régression est de 0,171 ; il exprime qu'une augmentation de $n_{v}$ de 10-4 entraîne une augmentation relative du résultat Milko-Tester de 0,17 p. 100 (1).

Si nous prenons, à titre d'exemple, les échantillons de lait que nous avons analysés et dont l'indice de réfraction varie de $45.10^{-4}$, cela représente en moyenne une amplitude des écarts, par rapport à la méthode Röse-Gottlieb, de $\pm 1,3 \mathrm{~g} / \mathrm{kg}$ environ (pour un lait moyen de $34,0 \mathrm{~g} / \mathrm{kg}$ de matière grasse).

Nos résultats se rapprochent de ceux de Walstra (1965 b) qui donne pour une variation de 0,005 de la différence des indices de réfraction entre la graisse et le sérum, une différence de la mesure de turbidité de 6 p. 100 (soit 0,12 p. 100 pour une variation de 10de l'indice de réfraction).

Le coefficient de corrélation entre l'indice de réfraction et les différences relatives est de $0,87 \pm 0,10$. Cela signifie que les variations de l'indice de réfraction de la matière grasse expliquent, en moyenne, 76 p. 100 des différences observées entre le Milko-Tester et la méthode de référence.

(1) Remarque : Ce résultat a été confirmé en dosant au Milko-Tester des émulsions de triglycérides purs (tributyrine, trioléine) en concentration connue dans du lait écrémé. Nous avons observé qu'une augmentation de $10^{-4}$ de l'indice de réfraction de l'émulsion entraîne une augmentation de 0,21 p. 100 de la lecture au Milko-Tester. 


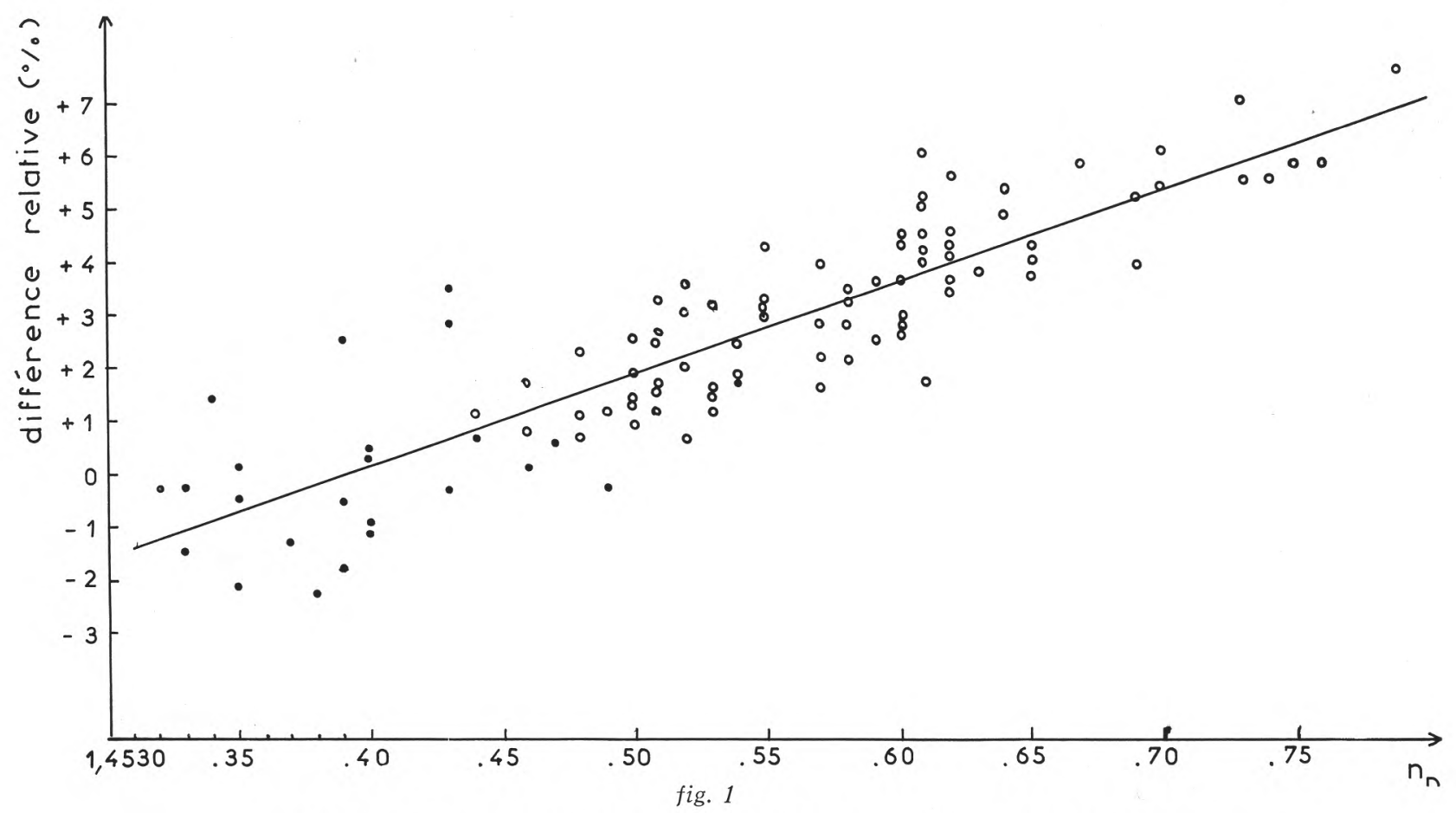

Relation entre l'indice de réfraction $\left(\eta_{\mathrm{D}}\right)$ de la matière grasse de 104 laits individuels et les différences relatives (p. 100) entre les dosages de matière grasse par le Milko-Tester et par la méthode de référence Röse-Gottlieb.

- : Prélèvements de mars à avril (avant la mise à l'herbe).

0 : Prélèvements de mai à juillet (après la mise à l'herbe). 
En conclusion, ces résultats montrent :

$1^{\circ}$ qu'il existe une relation linéaire entre l'indice de réfraction de la graisse du lait et la précision des dosages par le Milko-Tester,

$2^{\circ}$ que cette précision dépend essentiellement (soit 76 p. 100 de la variance totale) des variations de l'indice de réfraction. Par conséquent, la connaissance des facteurs de variation de l'indice de réfraction et de l'amplitude de ces variations doit fournir des indications sur la précision de l'appareil. On sait en particulier que la mise à l'herbe (figure 1) et plus généralement la saison, entraînent une modification de cet indice ; mais d'autres facteurs, comme l'alimentation ou l'individu jouent certainement un rôle important. Aussi, dans une étude en cours, essayons-nous de déterminer l'origine et l'amplitude des variations de l'indice de réfraction de la matière grasse du lait ainsi que leurs conséquences sur la précision des dosages de matière grasse avec le Milko-Tester.

\section{R és u m é}

Dans une étude portant sur 104 laits individuels prélevés entre le mois de mars et le mois de juillet, nous avons montré qu'il existe une corrélation $(r=+0,87)$ entre l'indice de réfraction de la graisse du lait et la précision des dosages de matière grasse avec l'appareil Milko-Tester (M K III).

Une augmentation de 10-4 de $\mathrm{n}_{\mathrm{n}}$, entraîne une augmentation relative de 0,17 p. 100 du taux de matière grasse déterminé par le Milko-Tester (par rapport à la méthode Röse-Gottlieb).

\section{S u m m a ry}

On 104 individual milks sampled from March to July, we have shown that there is a correlation $(r=+0,87)$ between the refractive index of milk fat and the accuracy of the fat content given by the Milko-Tester (M K III).

An increase of $n_{\mathrm{v}}$ of $10-4$ produces a relative increase of the Milko-Tester reading of 0,17 p. 100 (compared to Röse-Gottlieb results).

Reçu pour publication en septembre 1970.

\section{Références bibliographiques}

- Grappin (R.) et Jeunet (R.) (1970). - Essais de l'appareil « Milko-Tester Automatique "destiné au dosage en série de la matière grasse du lait. Le Lait, 50, 233-56.

- Haugaard (G.) and Pettinati (J. O.) (1959). - Photometric milk fat determination. J. Dairy Sci., 42, 1255-75.

- Mulder (H.) (1940). - Méthode rapide pour séparer la matière grasse du lait dans le but de déterminer l'indice de réfraction et d'autres propriétés de cette matière grasse. Verslag. Landb. Ouderzvek, 46, 505-14. Réf. Le Lait, $1942,22,159$.

- Pont (E. G.) (1955). - A de-emulsification technique for use in the peroyxyde test on the fat of milk, cream concentrated and dried milks. Aust. J. Dairy Techn., $\mathrm{n}^{\circ}$ mai-juin, 72.

- Walstra (P.) (1965 a). - Light scattering by milk fat globules. Neth. Milk Dairy J., 1965, 19, 93-109.

- Walstra (P.) (1965 b). - Turbidimetric determination of the fat content of milk. Neth. Milk Dairy J., 1965, 19, 266-82. 